\title{
COMPLETENESS, THE RECURSION THEOREM, AND EFFECTIVELY SIMPLE SETS
}

\author{
DONALD A. MARTIN
}

1. Introduction. The main result of this note is that every effectively simple set is complete, i.e., of the highest recursively enumerable degree of unsolvability. A recursively enumerable set of natural numbers is called simple if its complement, though infinite, possesses no infinite recursive subset. Let $W_{0}, W_{1}, \cdots$ be the standard enumeration of all recursively enumerable (r.e.) sets. Smullyan [8] calls an r.e. set $S$ effectively simple if $\bar{S}$ (the complement of $S$ ) is infinite and there is a recursive function $f$ such that, for every number $e$, if $W_{e} \subseteq \bar{S}$ then $f(e)$ is greater than the cardinality of $W_{e}$. It is immediate that every effectively simple set is simple. In [6] Sacks shows by direct construction that not every simple set is effectively simple. Sacks' theorem is a consequence of our main result and Friedberg's solution of Post's problem since [1] every nonzero r.e. degree of unsolvability is the degree of a simple set.

McLaughlin [2] calls an r.e. set with an infinite complement (a coinfinite r.e. set) $S$ strongly effectively simple if there is a recursive function $f$ such that, for each $e$, if $W_{e} \subseteq \bar{S}$ then $f(e)$ is greater than every member of $W_{e}$. Strongly effectively simple sets are all effectively simple, but we can, by varying slightly Sacks' construction and argument [6], produce an effectively simple set which is not strongly effectively simple. McLaughlin [2] proves that every strongly effectively simple set is either hypersimple or complete, a fact which is subsumed under our result.

The completeness of effectively simple sets can be proved by a simple argument using the recursion theorem, but we prefer to attack a more general problem. Several kinds of r.e. sets (e.g., creative sets [4] and quasicreative sets [7]) have been proved complete by methods which involve the recursion theorem. Our aim is to capture the essence of these methods. To do this, we shall define a rather general class $D$ of r.e. sets. It will be almost trivial to show-directly from definitions - that such classes as those of creative sets, quasicreative sets, and effectively simple sets are included in $D$. We shall use the recursion theorem to show that every member of $D$ is complete. We shall conclude by showing that every truth-table complete set belongs to $D^{1}{ }^{1}$

Received by the editors November 18, 1965.

1 A. H. Lachlan has pointed out to the author that, by replacing $h$ with a partial recursive functional in an appropriate manner, we could make $D$ into the class of all complete r.e. sets. 
2. The class D. Let $\beta_{0}, \beta_{1}, \cdots$ be the standard enumeration of all partial recursive functions (of one argument). We define a partial recursive function $\pi(e, z)$ as follows:

$\pi(e, z)=\mu y\left[(x)_{x \leq y}\left(\beta_{e}(x)\right.\right.$ is defined $)$ and there are at least $z$ numbers

$$
\left.x \leqq y \text { for which } \beta_{e}(x)=1\right] .
$$

The representing function of a set of numbers $A$ is that function which is zero for all $x \in A$ and 1 for all $x \notin A$. We call a relation $P(x, A, B)$ between numbers $x$, sets $A$, and sets $B$ admissible if there are recursive functions $g(e, x)$ and $h(x)$ with the following property: For all numbers $e$ and $x$ and for every set $C$, if $\pi(e, h(x))$ is defined and if the representing function of $C$ agrees with $\beta_{e}$ on all numbers $\leqq \pi(e, h(x))$, then

$$
\sim P\left(x, W_{g(e, x)}, C\right)
$$

where " " means not.

The property of admissible relations $P(x, A, B)$ which is crucial for the theorem below is, in intuitive terms, this: Given a number $x$ and a coinfinite recursive set $C$, we can effectively find the index of an r.e. set $W$ such that $\sim P(x, W, C)$, and furthermore we have an effective bound $h(x)$ to the information about $C$ which we will need to enumerate $W$.

We say that a relation $P(x, A, B)$ is satisfied by a set $C$ if

$$
\text { (n) } P\left(n, W_{n}, C\right) \text {. }
$$

We are now ready to define $D$. $D$ is to consist of those coinfinite r.e. sets $D$ such that some admissible relation is satisfied by $D$.

ThEOREM. Every member of $D$ is complete.

Proof. Let $D$ belong to $D$. Let $P(x, A, B)$ be an admissible relation satisfied by $D$, and let $g(e, x)$ and $h(x)$ be the functions whose existence is guaranteed by the admissibility of $P$. Let $d$ be a recursive function whose range is $D$. For each $s$, we set $D^{s}=\left\{x:(E y)_{y \unlhd_{s}} d(y)\right.$ $=x\}$. For each $s$ and $x$, let $K_{x}^{s}$ be the set of the least $x$ members of $\overline{D^{s}}$.

There is a recursive function $p(e, t)$ such that, for each $e$ and $t$,

$$
\beta_{p(e, t)}=\left\{\begin{array}{l}
\text { the representing function of } D^{\beta_{e}(t)} \text { if } \beta_{e}(t) \text { is defined; } \\
\text { the empty partial function otherwise. }
\end{array}\right.
$$

By the recursion theorem, there is a recursive function $q(e, t)$ such that

$$
(e)(t)\left[W_{g(p(e, t), q(e, t))}=W_{q(e, t)}\right] .
$$


For each $e$ and $t$, let

$$
v(e, t)=\mu s\left[K_{h(q(e, t))}^{s} \subseteq \bar{D}\right] .
$$

$v$ is recursive in $D$.

Let $W$ be any r.e. set. Let $f(s, x)$ be a recursive function such that

$$
(x)[x \in W \leftrightarrow(E s) f(s, x)=0] .
$$

Let $e$ be an index of the partial recursive function $\mu s[f(s, x)=0]$. We shall show that $\beta_{e}(t)<v(e, t)$ for each $t$ such that $\beta_{e}(t)$ is defined. This will mean that $W$ is recursive in $v$ and hence in $D$, since we shall have

$$
(x)\left[x \in W \leftrightarrow(E s)_{s<v(e, x)} f(s, x)=0\right] .
$$

Suppose that, contrary to what we wish to establish, $\beta_{e}(t)$ is defined for some $t$ and $\beta_{e}(t) \geqq v(e, t)$. Then, by the definitions of $v$ and of $K_{x}^{s}$,

$$
K_{h(q(e, t))}^{\beta e(t)} \subseteq \bar{D} .
$$

But this just says that the least $h(q(e, t))$ members of $\left(D^{\beta_{\boldsymbol{\theta}}(t)}\right)$ - are in $\bar{D}$. Hence the representing function of $D$ agrees with $\beta_{p(e, t)}$, the representing function of $D^{\beta_{e}(t)}$, on all numbers $\leqq \pi(p(e, t), h(q(e, t)))$. But then

$$
\sim P\left(q(e, t), W_{o(p(e, t), q(e, t))}, D\right),
$$

and the definition of $q(e, t)$ gives us that

$$
\sim P\left(q(e, t), W_{q(e, t)}, D\right),
$$

which contradicts the hypothesis that $P$ is satisfied by $D$.

3. Effectively simple sets. We now show that every effectively simple set belongs to $D$. Let $S$ be effectively simple and let $f$ be a recursive function such that, for each $e$, if $W_{e} \subseteq \bar{S}$ then $f(e)$ is greater than the cardinality of $W_{e}$. Let $h(x)=f(x)$, for all $x$. Let $g(e, x)$ be a recursive function satisfying

$$
y \in W_{o(e, x)} \leftrightarrow \pi(e, h(x)) \text { is defined and } y \leqq \pi(e, h(x)) \text { and } \beta_{e}(y)=1 \text {. }
$$

Let $P(x, A, B)$ hold if and only if $h(x)$ is greater than the cardinality of $A$ or $A \Phi \bar{B}$.

Evidently $P$ is satisfied by $S$. Using $h$ and $g$, we can see that $P$ is admissible, as follows:

Let $x$ and $e$ be such that $\pi(e, h(x))$ is defined and let $C$ be a set whose representing function agrees with $\beta_{e}$ on all numbers $\leqq \pi(e, h(x))$. 
Since $\pi(e, h(x))$ is defined, $W_{g(e, x)}$ is the set of all numbers $\leqq \pi(e, h(x))$ which belong to $\bar{C}$. Hence $W_{g(e, x)} \subseteq \bar{C}$. Furthermore, $W_{g(e, x)}$ has $h(x)=f(x)$ members, so that $\sim P\left(x, W_{g(e, x)}, C\right)$.

In [8] Smullyan proves a theorem one of whose main consequences is the existence of hypersimple effectively simple sets. As a final remark on effectively simple sets, we point out that this consequence follows from general elementary facts: Evidently every coinfinite r.e. superset of an effectively simple set is effectively simple; and in [3] it is shown that every simple set has a hypersimple superset. Similar considerations show that there are hypersimple strongly effectively simple sets.

4. Truth-table complete sets. The truth-table complete sets were defined by Post [5] and are a proper subclass of the complete sets. For any function $v$, the function $\tilde{v}$ is defined by $\tilde{v}(x)=\prod_{i<x} p_{i}^{o(t)}$, where $p_{i}$ is the $i+1$ st prime number. An r.e. set $V$ is truth-table complete if, for every r.e. set $W$, there are recursive functions $p(x, y)$ and $q(x)$ such that, for each $x, p(x, \tilde{v}(q(x)))$ is the representing function of $W$, where $v$ is the representing function of $V$.

TheOREM. Every truth-table complete set is a member of $D$.

Proof. Let $V$ be truth-table complete, and let $v$ be its representing function. Let $W=\left\{x: x \in W_{x}\right\}$. Let $p$ and $q$ be recursive functions related to $V$ and $W$ as in the definition of truth-table completeness. Let

$$
\begin{aligned}
& P(x, A, B) \leftrightarrow[x \in A \leftrightarrow p(x, \tilde{b}(q(x)))=0], \text { where } b \text { is the representing } \\
& \text { function of } B ; \\
& h(x)=q(x) ; \\
& y \in W_{g(e, x)} \leftrightarrow p\left(y, \tilde{\beta}_{e}(q(y))\right)>0,
\end{aligned}
$$

with $g$ recursive. Clearly $P$ is satisfied by $V$. The reader may easily verify the admissibility of $P$, using $h$ and $g$.

It is worth noting that not every member of $D$ is truth-table complete. In particular, Post [5] shows that no hypersimple set is truthtable complete, whereas we have seen in $\$ 3$ that there are hypersimple sets which belong to $D$.

\section{REFERENCES}

1. J. C. E. Dekker, $A$ theorem on hypersimple sets, Proc. Amer. Math. Soc. 5 (1954), 791-796.

2. T. G. McLaughlin, On a class of complete sets, Canad. Math. Bull. 8 (1965), 33-37. 
3. D. A. Martin, A theorem on hyperhypersimple sets, J. Symbolic Logic 28 (1963), 273-278.

4. J. Myhill, Creative sets, Z. Math. Logik. Grundlagen Math. 1 (1955), 97-108.

5. E. Post, Recursively enumerable sets of postivie integers and their decision problems, Bull. Amer. Math. Soc. 50 (1944), 284-316.

6. G. E. Sacks, A simple set which is not effectively simple, Proc. Amer. Math. Soc. 15 (1964), 51-55.

7. J. R. Schoenfield, Quasicreative sets, Proc. Amer. Math. Soc. 8 (1957), 964-967.

8. R. M. Smullyan, Effectively simple sets, Proc. Amer. Math. Soc. 15 (1964), 893-894.

\section{HARVARD UNIVERSITY}

